\title{
QUALIDADE DA SILAGEM DE RESÍDUO DE MANGA COM DIFERENTES ADITIVOS ${ }^{1}$
}

\author{
Quality of mango residue silage with different additives
}

\author{
Cristovão Colombo de Carvalho Couto Filho², José Cleto da Silva Filho ${ }^{3}$, Arnaldo Prata Neiva Júnior ${ }^{4}$, \\ Rilke Tadeu Fonseca de Freitas ${ }^{3}$, Ronan Magalhães de Souza ${ }^{4}$, José Airton Rodrigues Nunes ${ }^{5}$
}

\begin{abstract}
RESUMO
Com o objetivo de avaliar a qualidade da silagem de resíduo de manga (Mangifera indica L.) em mistura com diferentes aditivos, em níveis crescentes de adição, foi conduzido este estudo. O delineamento experimental utilizado foi o inteiramente casualizado, com três repetições, sendo os tratamentos dispostos em um esquema fatorial do tipo $(4 \times 3)+1$, sendo quatro aditivos (milho desintegrado com palha e sabugo (MDPS), palha de feijão (PF), sabugo de milho (SM) e casca de café (CC)) em três níveis de adição $(10,20$ e $30 \%)$ e um tratamento testemunha (resíduo de manga puro ensilado). O material experimental foi ensilado em silos de PVC adaptados com válvula tipo "Bunsen" com capacidade para aproximadamente $3 \mathrm{~kg}$ cada. Todos os aditivos utilizados elevaram os teores de MS. À medida que se aumentou os níveis de adição dos aditivos, houve uma redução nos valores de poder tampão (PT). Os valores de $\mathrm{pH}$ e nitrogênio amoniacal $\left(\mathrm{N}_{-} \mathrm{NH}_{3} / \% \mathrm{~N}\right.$ total) mantiveram-se dentro dos padrões indicativos de um bom processo fermentativo. O padrão de fermentação das silagens aditivadas foi satisfatório, sem haver comprometimento na qualidade destas. Os aditivos milho desintegrado com palha e sabugo, palha de feijão ou casca de café podem ser adicionados ao processo de ensilagem do resíduo de manga nos níveis de adição de 20 ou $30 \%$, melhorando seu padrão fermentativo.
\end{abstract}

Termos para indexação: Resíduos agroindustriais, Mangifera indica, aditivos, qualidade.

\section{ABSTRACT}

With the objective to evaluate the quality of mango (Mangifera indica L.) residue silage in mixture with different additives at growing levels of addition, this study was undertaken. The experimental design utilized was the completely randomized, with three replicates. The treatments were arranged in a factorial scheme $(4 \times 3)+1$ type and the additives being four (ground ears with husks $(\mathrm{GEWH})$, bean straw (BS), corn cob (CC) and coffee hull (CH)) at three levels of addition $(10,20$ and 30\%) and a control treatment (ensiled unmixed mango residue). The experimental material was ensiled in PVC silos, fitted with a "Bunsen" type valve, with a capacity of about $3 \mathrm{~kg}$ each. All the additives utilized raised the DM contents of the silages. As the levels of addition of the additives were increased, there was a reduction in the values of buffering power (PT). The values of $\mathrm{pH}$ and ammoniac nitrogen $\left(\mathrm{N}-\mathrm{NH}_{3} / \%\right.$ total $\mathrm{N}$ ) were maintained within the indicative standard of a goof fermentative process. The fermentation standard of silages with additives was satisfactory with no damage in their quality. The additives GEWH, BS or CH may be added to the process of ensiling the mango residue at the levels of addition of 20 or $30 \%$, to improve its fermentative standard for silages of good quality.

Index terms: Agroindustrial residue, Mangifera indica, additives, silage quality.

(Recebido em 19 de abril de 2005 e aprovado em 31 de maio de 2006)

\section{INTRODUÇÃO}

O processamento industrial de produtos agrícolas no Brasil, para a extração de sucos, polpas, óleos e molhos, gera uma grande quantidade de subprodutos, constituídos principalmente por sementes, cascas e polpa. O beneficiamento da manga, por sua vez, produz uma quantidade de resíduos que corresponde, aproximadamente, de 28 a $43 \%$ do total da fruta (NATIVIDAD-FERRER, 1987). No cenário nacional, a Região Sudeste ocupa o segundo lugar na produção de manga, ao passo que o Estado de Minas Gerais é o segundo maior produtor, com aproximadamente 35 mil toneladas (AGRIANUAL, 2004).
Em virtude do Brasil ser também um país marcado pela estacionalidade da produção de forragens, fator que constitui o principal entrave na exploração pecuária nacional e mundial, busca-se, através de técnicas de conservação de alimento, a obtenção de índices produtivos satisfatórios. Nesse contexto, é indiscutível o papel da silagem como volumoso suplementar na alimentação de ruminantes em períodos de escassez de forragem.

A ensilagem de resíduos provenientes da agroindústria frutícola vem surgindo como uma alternativa às culturas tradicionais, tendo como vantagem um baixo custo de aquisição do material a ser ensilado. A utilização destes resíduos na forma de silagem, além de constituir

${ }^{1}$ Parte da Dissertação de Mestrado do primeiro autor

${ }^{2}$ MSc. em Zootecnia - Universidade Federal de Lavras/UFLA - Cx. P. 3037 - 37.200-000 - Lavras, MG - coutofil@yahoo.com.br

${ }^{3}$ Professor do Departamento de Zootecnia/DZO - Universidade Federal de Lavras/UFLA - Cx. P. 3037 - 37.200-000 - Lavras, MG

${ }^{4}$ Doutorando em Zootecnia - Universidade Federal de Lavras/UFLA - Cx. P. 3037 - 37.200-000 - Lavras, MG.

${ }^{5}$ MSc. em Estatística e Experimentação Agropecuária - Universidade Federal de Lavras/UFLA- Cx. P. 3037 - 37.200-000 - Lavras, MG. 
uma forma de aproveitamento de um material que seria desperdiçado, contribui para minimizar o impacto causado pelo acúmulo destes resíduos no meio ambiente. O resíduo de processamento da manga, no entanto, apresenta um alto teor de umidade, característica que interfere negativamente no processo fermentativo por permitir o surgimento de fermentações secundárias indesejáveis, levando à queda na qualidade da silagem e a perdas por drenagem.

O termo "qualidade da silagem" é usado para descrever até que ponto o processo fermentativo ocorreu de maneira desejável, sendo que a determinação do índice $\mathrm{pH}$ e da concentração de nitrogênio amoniacal configuramse como indicadores adequados na avaliação deste processo (BREIREM \& ULVESLI, 1960). Existem, no entanto, características que, segundo McCullough (1977) e McDonald et al. (1991), irão influenciar de maneira decisiva na natureza da fermentação e na conservação da massa ensilada, podendo-se destacar os teores de umidade e de carboidratos solúveis, bem como o poder tampão do material a ser ensilado. Entre as alternativas que podem ser utilizadas para obtenção de silagens de melhor qualidade pode-se citar o uso de aditivos absorventes da umidade.

Com o objetivo de avaliar a qualidade do resíduo de manga (Mangifera indica L.) ensilado com diferentes aditivos, em níveis crescentes de adição, foi conduzido este estudo.

\section{MATERIAL E MÉTODOS}

O trabalho foi conduzido nas dependências do Departamento de Zootecnia da Universidade Federal de Lavras (DZO /UFLA), Lavras - MG. O delineamento experimental foi o inteiramente casualizado (DIC), com 13 tratamentos e três repetições. Os tratamentos foram dispostos em um esquema fatorial com tratamento adicional do tipo $(4 \times 3)+1$, quatro aditivos (milho desintegrado com palha e sabugo - MDPS, palha de feijão - PF, sabugo de milho - SM e casca de café - CC), três níveis de utilização $(10,20$ e $30 \%)$, com um tratamento testemunha (resíduo de manga puro ensilado). O resíduo de processamento da manga foi concedido pela empresa Bela Ischia, situada no município de Astolfo Dutra-MG, e os aditivos milho desintegrado com palha e sabugo (MDPS), palha de feijão (PF), sabugo de milho (SM) e casca de café (CC) foram fornecidos por produtores dos municípios de Rio Pomba e Lavras, Minas Gerais.

O resíduo de manga, constituído por casca e polpa, foi ensilado da forma que foi obtido na indústria. Os aditivos passaram previamente por um processo de desintegração (espiga de milho integral, PF e CC) ou trituração (SM), sendo reduzidos a partículas de 0,5 a $1 \mathrm{~cm}$. O resíduo juntamente com os aditivos foram levados para um galpão, onde se realizou a ensilagem. Para tanto, foram utilizados silos de PVC com diâmetro de $10 \mathrm{~cm}$ e altura de $50 \mathrm{~cm}$, adaptados com válvula tipo "Bunsen", com capacidade para aproximadamente $3 \mathrm{~kg}$ de silagem, obtendo-se uma densidade de $600 \mathrm{~kg} / \mathrm{m}^{3}$.

$\mathrm{O}$ resíduo de manga destinado à produção da silagem testemunha foi imediatamente ensilado. Os tratamentos aditivados foram constituídos pela mistura do resíduo de manga com cada um dos diferentes aditivos nos níveis de adição de 10, 20 e 30\%. Para tanto, foram realizadas as pesagens nas devidas proporções, peso a peso, misturandose ambos no momento da ensilagem. Foram retiradas amostras dos materiais, antes e após a ensilagem, das quais uma parte foi conservada em freezer e a outra foi levada para uma estufa de ventilação forçada, na qual permaneceu a 65 ${ }^{\circ} \mathrm{C}$ por 120 horas, em virtude da alta umidade do material. Posteriormente, essas amostras foram moídas e armazenadas em potes plásticos devidamente identificados. A compactação dos materiais ensilados foi feita manualmente com auxílio de uma barra de ferro. Em seguida, estes foram fechados com tampas de PVC dotadas de válvula tipo "Bunsen" e vedados com fita crepe. Finalmente, os silos foram levados para um galpão, onde foram armazenados com a válvula voltada para baixo, para que o efluente que viesse a ser produzido pudesse ser eliminado, em temperatura ambiente e sob a proteção de luz solar e chuvas. Após 70 dias os silos foram abertos, sendo desprezadas as porções das extremidades de cada silagem. O material a ser analisado foi homogeneizado e amostrado, sendo parte acondicionada em sacos plásticos e conservada em freezer para análises posteriores. Foram retiradas amostras de $10 \mathrm{~g}$ de cada silo para se proceder à leitura do $\mathrm{pH}$ de imediato.

As análises do resíduo de manga, dos aditivos e das silagens foram realizadas no Laboratório de Pesquisa Animal do Departamento de Zootecnia da Universidade Federal de Lavras. A análise do teor de matéria seca (MS), foi realizada conforme recomendações da Association of Official Analytical Chemist (AOAC, 1990). Das amostras de silagens congeladas foi extraído o suco, com prensa hidráulica, para determinação do teor de nitrogênio amoniacal como porcentagem do nitrogênio total $\left[\mathrm{N}-\mathrm{NH}_{3}\right.$ (\% $\mathrm{N}$ total)], segundo método descrito (TOSI et al., 1975). As determinações do poder tampão foram feitas utilizando amostras do material original de acordo com a técnica descrita por Playne \& McDonald (1966). O pH foi determinado com a utilização de um potenciômetro (SILVA, 1998). 
Para efeito de análise estatística, os dados foram submetidos à análise de variância usando o procedimento GLM (General Linear Model) do software SAS (Statistical Analysis System) v. 8.0 (SAS INSTITUTE, 1999). O tratamento testemunha foi comparado com todos os tratamentos aditivados utilizando o teste de Dunnett a 5\% de probabilidade. Para estudo detalhado da interação aditivos por níveis de adição, foram realizados os desdobramentos adequados, estudando o efeito de aditivos em cada nível de adição, aplicando-se o teste de Scott-Knott (CRUZ, 2004) ao nível de 5\% de significância e, de outro modo, o efeito dos níveis de adição em cada aditivo por meio da análise de regressão linear simples.

\section{RESULTADOS E DISCUSSÃO}

O teor de matéria seca e os valores de poder tampão do resíduo de manga e dos aditivos utilizados na ensilagem encontram-se na Tabela 1.

O menor teor de MS da silagem de resíduo de manga $(16,84 \%)$ em relação ao material antes de ensilar (21,53\%), como se pode observar nas Tabelas 1 e 2, possivelmente estão relacionados à diminuição do conteúdo celular, principalmente de carboidratos solúveis durante o processo fermentativo (WOOLFORD, 1984) e perda de MS por meio de efluentes (MCDONALD et al., 1991).

Foi observada diferença significativa $(\mathrm{P}<0,05)$ na comparação do tratamento testemunha em relação aos aditivados, registrando-se elevação significativa nos teores de MS (Tabela 2). Todos os aditivos mostraram-se eficientes em aumentar o teor de MS do resíduo de manga. No entanto, o nível de adição de $10 \%$ mostrou-se insuficiente para a obtenção do percentual de 28 a $34 \%$ de MS, o qual, segundo McCullough (1977), é necessário para que haja um adequado processo fermentativo. Resultados similares foram obtidos por Santos (1995), que avaliando o valor nutritivo de silagens de resíduo de maracujá, constatou que a adição de $10 \%$ de CC ou PF não foi suficiente para elevar os teores de MS para níveis considerados satisfatórios.

O teor de matéria seca (MS) das silagens de resíduo de manga foi influenciado pelos aditivos $(\mathrm{P}<0,01)$, níveis de adição $(\mathrm{P}<0,01)$ e interação entre eles $(\mathrm{P}<0,05)$. Analisando o efeito de aditivo em cada nível de adição, observou-se efeito significativo $(\mathrm{P}<0,01)$ nos níveis de adição de 20 e $30 \%$. Dentre os aditivos utilizados, a PF foi o que promoveu maior elevação nos teores de matéria seca no nível de adição de $20 \%$, não diferindo do MDPS no nível de $30 \%(\mathrm{P}<0,05)$ (Tabela 2).

TABELA 1 - Médias dos teores de matéria seca e dos valores de poder tampão do resíduo de manga e dos aditivos utilizados na ensilagem.

\begin{tabular}{lccccc}
\hline \multirow{2}{*}{ Variável } & \multicolumn{3}{c}{ Alimento } \\
\cline { 2 - 6 } & $\mathbf{R M}^{1}$ & MDPS $^{2}$ & PF $^{3}$ & $\mathbf{S M}^{4}$ & CC $^{5}$ \\
\hline MS $(\%)^{6}$ & 21,53 & 88,54 & 84,68 & 85,95 & 84,13 \\
PT $^{7}$ & 29,74 & 2,46 & 3,35 & 1,34 & 12,15 \\
\hline
\end{tabular}

${ }^{1}$ Resíduo de manga (original); ${ }^{2}$ Milho desintegrado com palha e sabugo; ${ }^{3}$ Palha de feijão; ${ }^{4}$ Sabugo de milho; ${ }^{5}$ Casca de café; ${ }^{6} \mathrm{Com}$ base na matéria natural; ${ }^{7}$ Poder tampão (eq.mg NaOH /100 g MS).

TABELA 2 - Médias dos teores de matéria seca (MS) das silagens de resíduo de manga aditivadas com MDPS, PF, SM e CC nos diferentes níveis de adição.

\begin{tabular}{cccc}
\hline \multirow{2}{*}{ Aditivo } & \multicolumn{3}{c}{ Níveis de adição } \\
\cline { 2 - 4 } & $\mathbf{1 0 \%}$ & $\mathbf{2 0 \%}$ & $\mathbf{3 0 \%}$ \\
\hline MDPS & $21,96 * \mathrm{a}$ & $29,28 * \mathrm{~b}$ & $36,51 * \mathrm{a}$ \\
PF & $22,79 * \mathrm{a}$ & $31,96 * \mathrm{a}$ & $37,05 * \mathrm{a}$ \\
SM & $21,14 * \mathrm{a}$ & $26,85 * \mathrm{c}$ & $33,49 * \mathrm{~b}$ \\
CC & $23,64 * \mathrm{a}$ & $28,70 * \mathrm{~b}$ & $34,20 * \mathrm{~b}$ \\
\hline Testemunha $^{1}$ & & 16,84
\end{tabular}

\footnotetext{
* Diferem do tratamento testemunha pelo teste de Dunnett $(\mathrm{P}<0,05)$.

Médias seguidas de letras distintas na mesma coluna diferem entre si pelo teste de Scott-Knott $(\mathrm{P}<0,05)$.

${ }^{1}$ Resíduo de manga puro ensilado.
} 
Analisando os níveis de adição dentro de cada aditivo, observou-se efeito significativo $(\mathrm{P}<0,01)$ em todos os aditivos utilizados. Os teores de MS aumentaram linearmente à medida que foram elevados os níveis de adição dos aditivos (Figura 1).

O efeito obtido com a utilização de MDPS neste trabalho está em concordância com os resultados obtidos por Coan et al. (2002) e Santos (2004), que verificaram elevações nos teores de MS da silagem de cana-de-açúcar quando este aditivo foi utilizado, em comparação aos tratamentos testemunha, o que possibilitou uma melhor conservação da silagem. Com relação ao aditivo $\mathrm{CC}$, uma resposta linear positiva também foi relatada por Souza et al. (2003), que estudando a adição de níveis crescentes deste aditivo na ensilagem do capim-elefante, estimaram acréscimo de $0,54 \%$ no teor de MS por unidade de CC adicionada, valor este próximo ao obtido nesta pesquisa, cujo acréscimo foi de $0,53 \%$. Resultados semelhantes aos obtidos com o uso do SM nos níveis de 20 e $30 \%$ foram relatados por Tosi et al. (1999) ao fazerem uso do mesmo aditivo em níveis de 30 e $40 \%$ na ensilagem do capimelefante, obtendo teores de MS de 29,41 e 33,41\%, respectivamente.

Observou-se diferença significativa $(\mathrm{P}<0,05)$ nos valores de poder tampão de todos os tratamentos aditivados em relação ao tratamento testemunha (Tabela 3). Constatou-se que todos os aditivos, nos diferentes níveis de utilização, foram eficientes na redução do poder tampão do resíduo de manga sem aditivo $(29,74$ eq.mg NaOH /100 g MS).



FIGURA 1 - Teor de matéria seca (MS) das silagens de resíduo de manga em função dos níveis de adição dos aditivos.

TABELA 3 - Médias dos valores de poder tampão do resíduo de manga original aditivado com MDPS, PF, SM e CC nos diferentes níveis de adição.

\begin{tabular}{cccc}
\hline \multirow{2}{*}{ Aditivo } & \multicolumn{3}{c}{ Níveis de adição } \\
\cline { 2 - 4 } & $\mathbf{1 0 \%}$ & $\mathbf{2 0 \%}$ & $\mathbf{3 0 \%}$ \\
\hline MDPS & $20,35 * \mathrm{~b}$ & $14,85 * \mathrm{c}$ & $11,86 * \mathrm{c}$ \\
PF & $19,46 * \mathrm{c}$ & $14,68 * \mathrm{c}$ & $12,15 * \mathrm{c}$ \\
SM & $21,94 * \mathrm{a}$ & $17,53 * \mathrm{~b}$ & $14,77 * \mathrm{~b}$ \\
CC & $19,13 * \mathrm{c}$ & $21,06 * \mathrm{a}$ & $19,58 * \mathrm{a}$ \\
\hline Testemunha $^{1}$ & & 29,74 & \\
\hline
\end{tabular}

* Diferem do tratamento testemunha pelo teste de Dunnett $(\mathrm{P}<0,05)$.

Médias seguidas de letras distintas na mesma coluna diferem entre si pelo teste de Scott-Knott $(\mathrm{P}<0,05)$.

${ }^{1}$ Resíduo de manga puro ensilado. 
Os valores de poder tampão do material original foram influenciados $(\mathrm{P}<0,01)$ pelos aditivos, níveis de adição e interação entre eles. Analisando o efeito de aditivo em cada nível de adição, observou-se efeito significativo $(\mathrm{P}<0,01)$ em todos os níveis de adição. Dentre os aditivos utilizados, a PF foi o que promoveu maior redução nos valores de poder tampão nos diferentes níveis, não diferindo no entanto, do MDPS nos níveis de adição $(\mathrm{P}<0,05)$ de 20 e $30 \%$ (Tabela 3 ). Nestes mesmos níveis de adição a CC foi o aditivo que registrou os maiores valores de poder tampão.

Analisando os níveis de adição dentro de cada aditivo, observou-se efeito significativo $(\mathrm{P}<0,01)$ de todos os aditivos utilizados. Foram observadas reduções lineares significativas $(\mathrm{P}<0,01)$ nos valores de poder tampão à medida que foram elevados os níveis de adição dos aditivos MDPS, PF e SM de $10 \%$ para $30 \%$, podendo-se assinalar reduções de 8,5, 7,3 e 7,2 unidades percentuais, respectivamente (Figura 2 ).

No aditivo CC foi observado comportamento distinto em relação aos demais, registrando-se um valor máximo observado, do poder tampão, de 21,06 eq.mg $\mathrm{NaOH} / 100$ g MS no nível de adição de 20\% (Tabela 3). Este fato deve-se, possivelmente, à concentração de potássio neste aditivo, elemento este que apresenta atividade tamponante (MCDONALD et al., 1991), e ao teor de PB $(10,17 \%)$ registrado nesse aditivo. Para Woolford (1984), as proteínas, dentre outros fatores, são responsáveis pela atividade tamponante das plantas, porém respondem por apenas 10 a $20 \%$ desta. Segundo Playne \& McDonald (1966), o poder tamponante devese, na maior parte (68 a 80\%) de sua magnitude, à fração ânion da forragem, representada pelos sais orgânicos, ortofosfatos, sulfatos, nitratos e cloretos.

Efeito semelhante ao observado no estudo com a utilização do SM na ensilagem do resíduo de manga foram relatados por Tosi et al. (1995, 1999). Esses pesquisadores concluíram que apenas o SM, dentre todos os tratamentos utilizados na ensilagem do capim-elefante, foi eficiente na redução da capacidade tamponante dos materiais ensilados.

Em todos os tratamentos aditivados, independentemente do aditivo ou nível de utilização dos mesmos, os valores de poder tampão se mostraram inferiores ao valor de 22,63 eq.mg $\mathrm{NaOH} / 100 \mathrm{~g}$ MS observado por Tosi et al. (1975) na silagem de milho, considerada espécie forrageira que apresenta baixa capacidade tamponante.

Foi observada diferença significativa $(\mathrm{P}<0,05)$ nos valores de $\mathrm{pH}$ entre a silagem testemunha e as aditivadas, com exceção daquelas em que se fez uso dos aditivos MDPS e SM nos níveis de 10 e $20 \%$, cujos valores observados foram semelhantes (Tabela 4).

Pode-se constatar (Tabela 4) que apesar do leve aumento nos valores de $\mathrm{pH}$ das silagens aditivadas em relação à testemunha, que apresentou $\mathrm{pH}$ mais baixo $(3,30)$, esses valores encontram-se abaixo do $\mathrm{pH}$ limite de 4,2, os quais, segundo Woolford (1984), são indicativos de processo fermentativo adequado.



$\longrightarrow$ MDPS $\longrightarrow$ PF $\longrightarrow$ SM

FIGURA 2 - Valores de poder tampão (eq.mg NaOH /100 g MS) do resíduo de manga em função dos níveis de adição dos aditivos MDPS, PF e SM. 
Os valores de $\mathrm{pH}$ das silagens de resíduo de manga foram influenciados $(\mathrm{P}<0,01)$ pelos aditivos, níveis de utilização e interação entre eles. Analisando o efeito de aditivo em cada nível de adição, observou-se efeito significativo $(\mathrm{P}<0,01)$ em todos os níveis de adição. Como pode-se observar, o aditivo $\mathrm{CC}$ foi o que resultou em maior elevação do $\mathrm{pH}$ nos diferentes níveis de adição $(\mathrm{P}<0,05)$ (Tabela 4$)$. Este fato se deve, possivelmente, à elevada capacidade tamponante da CC (12,1 eq.mg NaOH /100 g MS) em relação aos demais aditivos (Tabela 1$)$.

Analisando-se os níveis de adição dentro de cada aditivo, foi observado $(\mathrm{P}<0,01)$ efeito significativo de todos os aditivos utilizados. Foi observado aumento linear significativo $(\mathrm{P}<0,01)$ nos valores de $\mathrm{pH}$ à medida que aumentaram os níveis de aditivos. Os valores médios estimados de $\mathrm{pH}$ foram de 3,46, 3,62, 3,43 e 3,75, respectivamente nas silagens com MDPS, PF, SM e CC no nível de adição de 30\% (Figura 3).

Observou-se diferença significativa $(\mathrm{P}<0,05)$ nos teores de nitrogênio amoniacal $\quad\left(\mathrm{N}-\mathrm{NH}_{3} / \% \mathrm{~N}\right.$ total $) \mathrm{da}$ silagem testemunha em relação às aditivadas (Tabela 5). Constatou-se também que, apesar do leve aumento desses teores nas silagens aditivadas em relação à testemunha, todos os valores obtidos permitem classificar as silagens estudadas, segundo McDonald et al. (1991), como muito boas, uma vez que os valores obtidos neste parâmetro foram inferiores a $10 \%$.

TABELA 4 - Médias dos valores de pH das silagens de resíduo de manga aditivadas com MDPS, PF, SM e CC nos diferentes níveis de adição.

\begin{tabular}{cccc}
\hline \multirow{2}{*}{ Aditivo } & \multicolumn{3}{c}{ Níveis de adição } \\
\cline { 2 - 4 } & $\mathbf{1 0 \%}$ & $\mathbf{2 0 \%}$ & $\mathbf{3 0 \%}$ \\
\hline MDPS & $3,35 \mathrm{c}$ & $3,36 \mathrm{~b}$ & $3,48 * \mathrm{c}$ \\
PF & $3,43 * \mathrm{~b}$ & $3,59 * \mathrm{a}$ & $3,59 * \mathrm{~b}$ \\
SM & $3,32 \mathrm{c}$ & $3,36 \mathrm{~b}$ & $3,43 * \mathrm{c}$ \\
CC & $3,53 * \mathrm{a}$ & $3,64 * \mathrm{a}$ & $3,74 * \mathrm{a}$ \\
\hline Testemunha $^{1}$ & & 3,30 & \\
\hline
\end{tabular}

* Diferem do tratamento testemunha pelo teste de Dunnett $(\mathrm{P}<0,05)$.

Médias seguidas de letras distintas na mesma coluna diferem entre si pelo teste de Scott-Knott $(\mathrm{P}<0,05)$.

${ }^{1}$ Resíduo de manga puro ensilado.

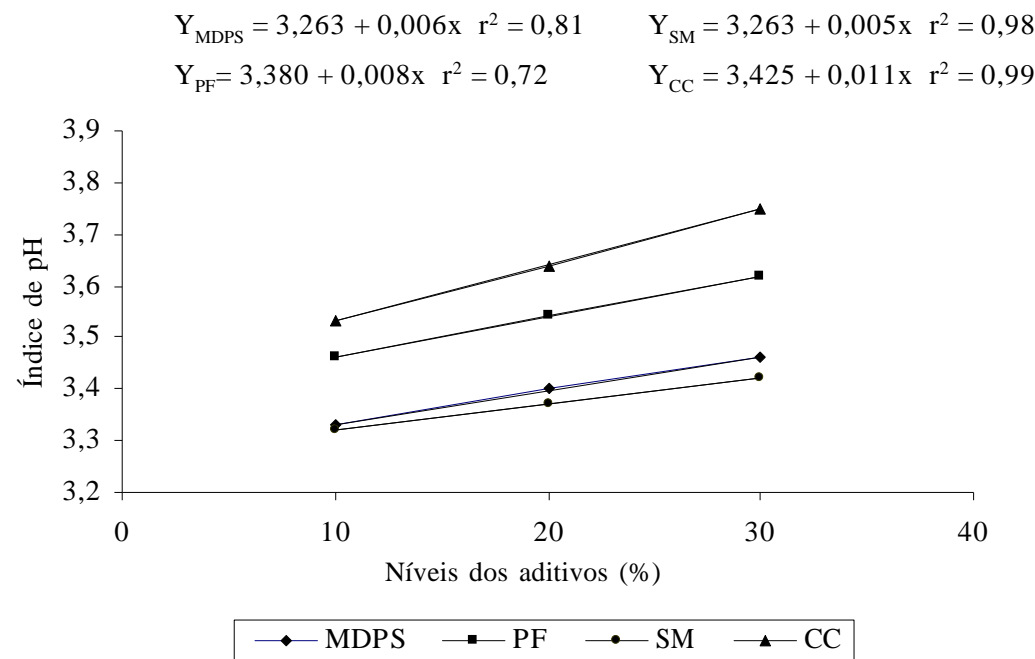

FIGURA 3 - Índice de pH das silagens de resíduo de manga em função dos níveis de adição dos aditivos. 
Os teores de $\mathrm{N}-\mathrm{NH}_{3}(\% \mathrm{~N}$ total) das silagens de resíduo de manga foram influenciados $(\mathrm{P}<0,01)$ pelos aditivos, níveis de adição e interação entre eles. Analisando o efeito de aditivo em cada nível de adição, observou-se efeito significativo $(\mathrm{P}<0,01)$ nos níveis de adição de 20 e $30 \%$, sendo o aditivo CC o que permitiu a obtenção de silagens com menor teor de nitrogênio amoniacal $\left(\mathrm{N}^{-\mathrm{NH}_{3}} /\right.$ $\% \mathrm{~N}$ total) nestes níveis de adição $(\mathrm{P}<0,05)$ (Tabela 5). O efeito dos níveis de adição dentro de cada aditivo foi significativo $(\mathrm{P}<0,01)$ em todos os aditivos. Os teores de $\mathrm{N}-\mathrm{NH}_{3}(\% \mathrm{~N}$ total $)$ aumentaram linearmente à medida que foram elevados os níveis de adição dos aditivos (Figura 4). Resultados semelhantes aos obtidos com o aditivo SM foram relatados por Tosi et al. (1999). Estes autores registraram elevação nos teores de $\mathrm{N}-\mathrm{NH}_{3}(\% \mathrm{~N}$ total $)$ quando utilizaram este mesmo aditivo nos níveis de 30 e $40 \%$ na ensilagem de capim-elefante, em relação ao tratamento controle sem aditivo.

Segundo Woolford (1984), a proteólise se estende durante a fermentação quando não ocorrem condições ácidas suficientes para que os microrganismos indesejáveis sejam inibidos. Entretanto, nesta pesquisa, a amplitude de $\mathrm{pH}$ variou de 3,30 na silagem testemunha a 3,75, quando foram adicionados $30 \%$ de CC, mostrando-se suficiente para inibir a atividade proteolítica.

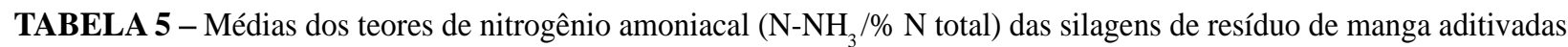
com MDPS, PF, SM e CC nos diferentes níveis de adição.

\begin{tabular}{cccc}
\hline \multirow{2}{*}{ Aditivo } & \multicolumn{3}{c}{ Níveis de adição } \\
\cline { 2 - 4 } & $\mathbf{1 0 \%}$ & $\mathbf{2 0 \%}$ & $\mathbf{3 0 \%}$ \\
\hline MDPS & $0,28 * \mathrm{a}$ & $0,42 * \mathrm{a}$ & $0,70 * \mathrm{a}$ \\
PF & $0,29 * \mathrm{a}$ & $0,40 * \mathrm{a}$ & $0,43 * \mathrm{c}$ \\
SM & $0,30 * \mathrm{a}$ & $0,42 * \mathrm{a}$ & $0,49 * \mathrm{~b}$ \\
CC & $0,23 * \mathrm{a}$ & $0,30 * \mathrm{~b}$ & $0,40 * \mathrm{c}$ \\
\hline Testemunha $^{1}$ & & 0,13 & \\
\hline
\end{tabular}

* Diferem do tratamento testemunha pelo teste de Dunnett $(\mathrm{P}<0,05)$.

Médias seguidas de letras distintas na mesma coluna diferem entre si pelo teste de Scott-Knott $(\mathrm{P}<0,05)$.

${ }^{1}$ Resíduo de manga puro ensilado.



FIGURA 4 - Teor de nitrogênio amoniacal $\left(\mathrm{N}-\mathrm{NH}_{3} / \% \mathrm{~N}\right.$ total) das silagens de resíduo de manga em função dos níveis de adição dos aditivos. 


\section{CONCLUSÕES}

O padrão de fermentação das silagens aditivadas foi satisfatório, sem haver comprometimento na qualidade fermentativa destas.

Os aditivos milho desintegrado com palha e sabugo, palha de feijão ou casca de café podem ser adicionados ao processo de ensilagem do resíduo de manga nos níveis de adição de 20 ou 30\%, melhorando seu padrão fermentativo.

\section{REFERÊNCIAS BIBLIOGRÁFICAS}

AGRIANUAL. Anuário da agricultura brasileira. São Paulo, 2004. 358 p.

ASSOCIATION OF OFICIAL ANALYTICAL CHEMIST. Official methods of analysis. 15. ed. Arlington, 1990. v. 1, 1117 p.

BREIREM, K.; ULVESLI, O. Ensiling methods. Herbage Abstracts, Fort Collins, v. 30, n. 1, p. 1-8, Jan. 1960.

COAN, R. M.; SILVEIRA, R. N.; BERNARDES, T. F. Composição química da cana-de-açúcar crua ou queimada ensilada com aditivo. In: REUNIÃO ANUAL DA SOCIEDADE BRASILEIRA DE ZOOTECNIA, 39., 2002, Recife. Anais... Recife: SBZ, 2002. CD-ROM.

CRUZ, C. D. Genes: programa para análise e processamento de dados baseado em modelos de genética e estatística experimental. Viçosa: UFV, 2004.

McCULlOUGH, M. E. Silage and silage fermentation. Feedstuffs, Mineapolis, v. 49, n. 13, p. 49-52, Mar. 1977.

McDONALD, P.; HENDERSON, A. R.; HERON, S. J. E. Biochemistry of silage. 2. ed. Marlow: Chalcombe, $1991.340 \mathrm{p}$.

NATIVIDAD FERRER, R. E. Avaliação das características de polpa de manga (Mangifera indica L.) para elaboração e armazenamento do néctar. 1987. Dissertação (Mestrado) - Universidade Federal de Viçosa, Viçosa, 1987.

PLAYNE, M. J.; McDONALD, P. The buffering constituints of herbage. Journal of Food Science and Agriculture, Barking, v. 17, n. 6, p. 264-268, June 1966.
SANTOS, M. A. S. Valor nutritivo de silagens de resíduo de maracujá (Passiflora edulis, Deuger), ou em mistura com casca de café (Coffea arabica, L.), bagaço de cana (Saccharum officinarum, L.) e palha de feijão (Phaseolus vulgaris, L.). 1995. 57 f. Dissertação (Mestrado) Universidade Federal de Lavras, Lavras, 1995.

SANTOS, R. V.Silagem de cana-de-açúcar em duas idades de corte com diferentes aditivos. 2004. 65 p. Dissertação (Mestrado) - Universidade Federal de Lavras, Lavras, 2004.

SAS INSTITUTE. SAS System for windows. Versão 8.0. Cary, 1999.

SILVA, D. J. Análise de alimentos: métodos químicos e biológicos. 2. ed. Viçosa: UFV, 1998. 166 p.

SOUZA, A. L.; BERNARDINO, F. S.; GARCIA, R. Valor nutritivo de silagem de capim-elefante (Pennisetum purpureum Schum) com diferentes níveis de casca de café. Revista Brasileira de Zootecnia, Viçosa, v. 32, n. 4, p. 828833, jul./ago. 2003.

TOSI, H.; RODRIGUES, L. R. A.; JOBIM, C. C.; OLIVEIRA, R. L.; SAMPAIO, A. A. M.; ROSA, B. Ensilagem do capimelefante cv. Mott sob diferentes tratamentos. Revista da Sociedade Brasileira de Zootecnia, Viçosa, v. 24, n. 6, p. 909-916, nov./dez. 1995.

TOSI, H.; SILVEIRA, A. C.; FARIA, V. P.; PEREIRA, R. L.Avaliação do girassol (helianthus annuus L.) como planta para ensilagem. Revista da Sociedade Brasileira de Zootecnia, Viçosa, v. 4, n. 1, p. 39-48, jan./fev. 1975.

TOSI, P.; MATTOS, W. R. S.; TOSI, H.; JOBIM, C. C.; LAVEZZO, W. Avaliação do capim-elefante (Pennisetum purpureum Schum.) cultivar Taiwan A-148, ensilado com diferentes técnicas de reduçãode umidade. Revista da Sociedade Brasileira de Zootecnia, Viçosa, v. 28, n. 5, p. 947-954, set./out. 1999.

WOOLFORD, M. K. The silage fermentation. New York: [s.n.], 1984. 305 p. 